\title{
Estimation of Some Lifetime Parameters of Flexible Reduced Logarithmic-Inverse Lomax Distribution under Progressive Type-II Censored Data
}

\author{
M. Mustafa Buzaridah $\left(\mathbb{D}\right.$, Dina A. Ramadan $\mathbb{D D}^{D}$, and B.S. El-Desouky $\mathbb{1}$ \\ Department of Mathematics, Faculty of Science, Mansoura University, Mansoura, Egypt \\ Correspondence should be addressed to Dina A. Ramadan; dina_ahmed2188@yahoo.com
}

Received 6 November 2021; Revised 13 January 2022; Accepted 20 January 2022; Published 25 February 2022

Academic Editor: Efthymios G. Tsionas

Copyright ( 2022 M. Mustafa Buzaridah et al. This is an open access article distributed under the Creative Commons Attribution License, which permits unrestricted use, distribution, and reproduction in any medium, provided the original work is properly cited.

\begin{abstract}
In this study, the estimation of parameters of a three-parameter flexible reduced logarithmic-inverse Lomax (FRL-IL) distribution based on progressive type-II right censored sample is studied. These methods include maximum likelihood estimations (MLEs) and Bayesian estimators. Approximate confidence intervals (ACIs) for the reliability and hazard functions are estimated based on the asymptotic distribution of maximum likelihood estimates (MLEs). In addition, two bootstrap CIs are also proposed. Bayesian estimates are obtained for symmetric and asymmetric loss functions such as squared error loss (SEL) and linear-exponential (LINEX) loss functions. The Gibbs within Metropolis-Hasting sampler procedure is applied using the Markov Chain Monte Carlo (MCMC) technique to get the Bayes estimates of the unknown parameters and their credible intervals (CRIs). Finally, a real-life dataset that represents a group of patients with bladder cancer is considered an application of the proposed methods.
\end{abstract}

\section{Introduction}

In life testing and reliability experiments, experiments must be often terminated before all units on the test have failed. For example, units may accidentally break in an industrial experiment, individuals may drop out of the study in a clinical trial, or the study may have to be terminated for lack of funds. For many reasons, the removal of units before failure is very often a procedure due to limitations of time and cost associated with the experiment. In such cases, in a lifetime test, it is impossible for experimenters to obtain the complete sample information in a short period of time. It leads us to the area of censoring, namely, some surviving units are removed from the experiment following a specific censoring scheme, and the final sample obtained is the censored sample, see Klein and Moeschberger [1]. Reducing the total test time and the associated test is one of the major reasons for censoring. In the life-testing experiment, if all the items under the test are observed until failure, the life test is said to be a complete life test. The available data in most practical situations are not complete. Therefore, censoring is very important in lifetime data analysis, either due to test time or budget constraints. Well-known censoring schemes are divided into two, type-I and type-II, censors. And then, a progressive censoring scheme is further proposed. This, in turn, allows the experimenters to remove the remaining units at different stages during the test, which is more flexible and realistic. The progressive type-II censoring scheme can be demonstrated as follows: the tester assigns independent and identical units on the life test. Suppose there are $n$ units to be tested at the beginning of the experiment, the lifetime test is terminated at the failure time of the $m$ th $(m<n)$ unit. Once the first failure occurs, time $t_{(1)}$ is registered, and $R_{1}$ units are randomly withdrawn from the remaining $n-1$ survival units. Consequently, time $t_{(2)}$ will be registered and $R_{2}$ units are randomly withdrawn from the remaining $n-R_{1}-2$ survival units at the occurrence of the second failure. This experiment stops at the $m^{\text {th }}$ failure, which is determined in advance, at time $t_{m}$, and $R_{m}=n-m-\sum_{i=1}^{m-1} R_{i}$ (Figure 1 ). 


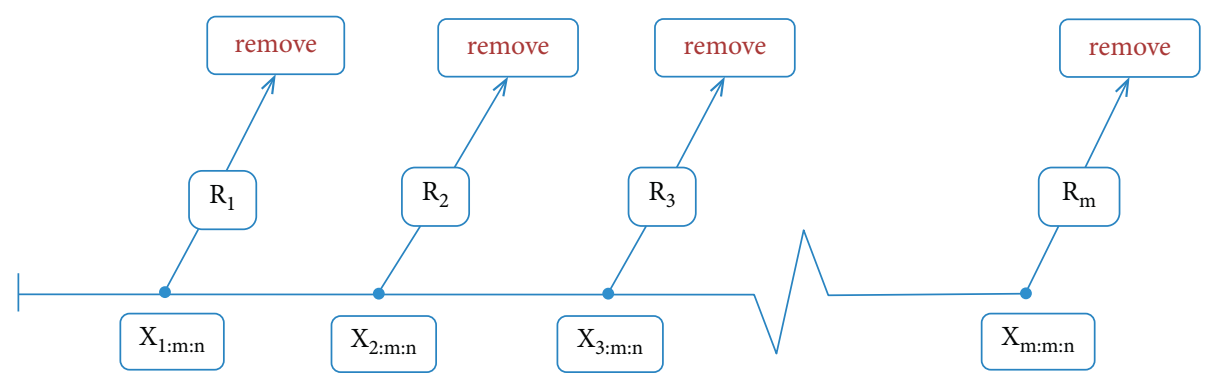

FIgURE 1: Type-II progressive censoring.

There are a lot of authors who have studied progressive type-II censoring with different breakdowns of failure time, including Mann et al. [2], Lawless [3], Meeker and Escobar [4], and Balakrishnan [5] studied the properties of progressively censored order statistics and provided an overview of various developments in inferential procedures based on progressive type-I and type-II right censored samples and identified some interesting possible problems for further research. Balakrishnan and Cramer [6] provide a comprehensive literature review on progressive censoring, details on this progressive censoring scheme, and its different applications. Tse et al. [7] indicated that, for example, the number of patients who drop out of a clinical test at each stage is random and cannot be predetermined. In some reliability trials, the experimenter may decide that it is inappropriate or dangerous to perform the test on some of the units tested even though these units did not fail. In such instances, the pattern of removal is random with each failure. For further information on the progressively censored samples, see Balakrishnan and Sandhu [8] and Balakrishnan and Aggarwala [9]. Aggarwala and Balakrishnan [10] have discussed inference for the case of progressive type II censored when lifetime distributions are Weibull, log normal, and exponential. Balakrishnan and Sandhu [11], and Aggarwala and Balakrishnan [12] have developed an algorithm to simulate general progressive type-II censored samples from uniform or any other continuous distributions. An interesting real application of progressive type II censored has been carried out by Montanari et al [13], and Eryilmaz and Bairamov [3] and also has been studying the estimation of parameters from different lifetime distributions based on progressive type-II censoring by many authors, including Balakrishnan and Kannan [14], Ali Mousa and Jaheen $[15,16]$, and Ali Mousa and Al-sagheer [17]. Recently, Salemi et al. [18] studied A-optimal and D-optimal censoring plans in progressive type-II right censored order statistics, Qin et al. [19] propose a new test statistic based on spacings to test whether the general progressive type-II censored samples are from an exponential distribution, Maiti and Kayal [20] studied estimation of the unknown parameters, reliability, and hazard functions of a log-logistic distribution under the progressive type-II censored sample, also, a study of the generalized Rayleigh distribution was presented when a controlled stepwise sample of the second type was available by Maiti and Kayal [21], and parametric (classical and the Bayes) point estimation procedures have been proposed to estimate the reliability characteristics, viz, reliability function and mean time to failure for XGD based on the complete observations, using four different estimation methods proposed by Saha and Yadav [22].

Bladder cancer is one of the most prevalent malignant tumors and one of the most expensive to treat per patient. Bladder cancer is chronically under-recognized as a public health concern and scientifically underfunded, despite its high prevalence, morbidity, death, and related management costs. In the last 30 years, 5-year survival rates for patients with prostate and kidney cancer have dramatically improved, but progress in bladder cancer has slowed a renewed interest from the clinical and research communities. Improved diagnostics, therapies, and health services for patients with bladder cancer will emerge as awareness and funding for the disease growth. For this reason, since our study will be on patients with bladder cancer, which requires us to reduce the number of experimental patients and the time of the lifetime experiment of these patients, we will apply the progressive type-II censoring scheme, which satisfies the requirements of obtaining good estimation by reducing the experimental time of patients and keeping some experimental patients from failure.

In this study, the three-parameter flexible reduced logarithmic-inverse Lomax (FRL-IL) distribution proposed by Buzaridah et al. [23] is considered as a good model for the failure times of the bladder cancer patient components. The random variable $X$ has a (FRL-IL) flexible reduced logarithmic-inverse Lomax distribution if its probability density function (PDF) and cumulative distribution function (CDF) are given by

$$
\begin{array}{r}
f(x ; \alpha, \beta, \lambda)=\frac{\left(\alpha \beta \lambda / x^{2}\right)[1+(\beta / x)]^{-(\alpha+1)}}{\left[1+\lambda-\lambda(1+(\beta / x))^{-\alpha}\right] \log [1+\lambda]}, \\
x>0, \alpha, \beta, \lambda>0, \\
F(x ; \alpha, \beta, \lambda)=1-\frac{\log \left[1+\lambda-\lambda(1+(\beta / x))^{-\alpha}\right]}{\log [1+\lambda]}, \\
x>0, \alpha, \beta, \lambda>0,
\end{array}
$$

while the reliability and hazard function of FRL-IL, respectively, are given by 


$$
\begin{aligned}
& S(x ; \alpha, \beta, \lambda)=\frac{\log \left[1+\lambda-\lambda(1+(\beta / x))^{-\alpha}\right]}{\log (1+\lambda)}, \quad x>0, \\
& h(x ; \alpha, \beta, \lambda)=\frac{\left(\alpha \beta \lambda / x^{2}\right)(1+(\beta / x))^{-(\alpha+1)}}{\left[1+\lambda-\lambda(1+(\beta / x))^{-\alpha}\right] \log \left[1+\lambda-\lambda(1+(\beta / x))^{-\alpha}\right]}, \quad x>0 .
\end{aligned}
$$

Buzaridah et al. [23] discussed the statistical properties for the FRL-IL, including the behavior of the function of probability density, the reliability or survival function, the function of the hazard rate, the function of the reversed hazard rate, and the residual (reversed) life. The moments and the moments generating function, quantile function, and skewness and kurtosis are obtained. In addition, they investigated the maximum likelihood estimators of the unknown parameters and their asymptotic confidence intervals based on complete data.

The rest of this study is organized as follows: In Section 2, the MLEs of $\alpha, \beta, \lambda, S(t)$, and $h(t)$ are formulated. Asymptotic confidence intervals based on the maximum likelihood estimates are presented in Section 3. The confidence intervals for the unknown parameters, reliability function, and hazard function are constructed using two parametric bootstrap procedures in Section 4. Bayes estimates for the aforementioned parameters and functions are also obtained for different loss functions such as squared error loss (SEL) and LINEX loss functions in Section 5. The real dataset has been analyzed in Section 6. A simulation study is performed in Section 7 to assess the quality of the different estimators developed in this study. Finally, we conclude the study in Section 8.

\section{Maximum Likelihood Estimators}

In this section, we study the estimation problem of the FRLIL parameters under progressive type-II censored samples using an estimation method called the maximum likelihood estimators (MLEs).

Let $X_{1: m: n}, X_{2: m: n}, \ldots, X_{m: m: n}, 1 \leq m \leq n$ denote the progressive type-II censored sample of size $m$ from a sample of $n$ with scheme $R_{1}, R_{2}, \ldots, R_{m}, m<n$ drawn from the FRLIL distribution. The likelihood function based on a progressive type-II censored sample from FRL-IL $(\alpha, \beta, \lambda)$ is given by

$$
\begin{aligned}
L(\alpha, \beta, \lambda \mid \underline{x})= & C \prod_{i=1}^{m} f\left(x_{i: m: n} ; \alpha, \beta, \lambda\right) \\
& \cdot\left[1-F\left(x_{i: m: n} ; \alpha, \beta, \lambda\right)\right]^{R_{i}}, \quad m<n,
\end{aligned}
$$

where $C$ is a constant defined as $C=n\left(n-1-R_{1}\right)(n-2-$ $\left.R_{1}-R_{2}\right), \ldots,\left(n-\sum_{i=1}^{m-1}\left(R_{i}+1\right)\right)$.

$F(\cdot)$ and $f(\cdot)$ are cdf and pdf defined in equations (1) and (2), respectively.

Substituting cdf and pdf into equation (4), then, we can write the log-likelihood function of $\alpha, \beta$ and $\lambda$ based on progressive type-II censored sample as follows:

$$
\begin{aligned}
& L(\alpha, \beta, \lambda \mid \underline{x})=C \prod_{i=1}^{m}\left(\frac{\left(\alpha \beta \lambda / x_{i: m: n}^{2}\right)\left[1+\left(\beta / x_{i: m: n}\right)\right]^{-(\alpha+1)}}{\left[1+\lambda-\lambda\left(1+\left(\beta / x_{i: m: n}\right)\right)^{-\alpha}\right] \log [1+\lambda]}\right) \times\left(\frac{\log \left[1+\lambda-\lambda\left(1+\left(\beta / x_{i: m: n}\right)\right)^{-\alpha}\right]}{\log [1+\lambda]}\right)^{R_{i}}, \\
& L(\alpha, \beta, \lambda \mid \underline{x})=C \times \alpha^{m} \beta^{m} \lambda^{m} \prod_{i=1}^{m} x_{i: m: n}^{-2} \frac{\prod_{i=1}^{m}\left[1+\left(\beta / x_{i: m: n}\right)\right]^{-(\alpha+1)}}{(\log [1+\lambda])^{m} \prod_{i=1}^{m}\left[1+\lambda-\lambda\left(1+\left(\beta / x_{i: m: n}\right)\right)^{-\alpha}\right] \times \frac{\prod_{i=1}^{m}\left(\log \left(1+\lambda-\lambda\left(1+\left(\beta / x_{i: m: n}\right)\right)^{-\alpha}\right)\right)^{R_{i}}}{\prod_{i=1}^{m}(\log (1+\lambda))^{R_{i}} .}}
\end{aligned}
$$

The log-likelihood function without the constant term can be written as

$$
\begin{aligned}
\ell= & m \log \alpha+m \log \beta+m \log \lambda-2 \sum_{i=1}^{m} \log x_{i: m: n}-(\alpha+1) \sum_{i=1}^{m} \log \left(1+\frac{\beta}{x_{i: m: n}}\right) \\
& -\sum_{i=1}^{m} \log \left(1+\lambda-\lambda\left(1+\frac{\beta}{x_{i: m: n}}\right)^{-\alpha}\right)-m \log (\log (1+\lambda)) \\
& +\sum_{i=1}^{m} R_{i} \log \left(\log \left(1+\lambda-\lambda\left(1+\frac{\beta}{x_{i: m: n}}\right)^{-\alpha}\right)\right)-\sum_{i=1}^{m} R_{i} \log (\log (1+\lambda)) .
\end{aligned}
$$


Calculating the first partial derivatives of $\ell$ with respect to $\alpha, \beta$, and $\lambda$ and equating each to zero, we get the likelihood equations as

$$
\begin{aligned}
\frac{\partial \ell}{\partial \alpha}= & \frac{m}{\alpha}-\sum_{i=1}^{m} \log \left(1+\frac{\beta}{x_{i: m: n}}\right)-\sum_{i=1}^{m} \frac{\lambda\left(1+\left(\beta / x_{i: m: n}\right)\right)^{-\alpha}\left(\ln \left(1+\left(\beta / x_{i: m: n}\right)\right)\right)}{\left(1+\lambda-\lambda\left(1+\left(\beta / x_{i: m: n}\right)\right)^{-\alpha}\right)} \\
& +\sum_{i=1}^{m} R_{i}\left[\frac{\lambda\left(1+\left(\beta / x_{i: m: n}\right)\right)^{-\alpha}\left(\ln \left(1+\left(\beta / x_{i: m: n}\right)\right)\right)}{\left(1+\lambda-\lambda\left(1+\left(\beta / x_{i: m: n}\right)\right)^{-\alpha}\right)\left(\ln \left(1+\lambda-\lambda\left(1+\left(\beta / x_{i: m: n}\right)\right)^{-\alpha}\right)\right)}\right] \\
\frac{\partial \ell}{\partial \beta}= & \frac{m}{\beta}-\sum_{i=1}^{m} \frac{\alpha+1}{\beta+x_{i: m: n}}-\sum_{i=1}^{m} \frac{\alpha \lambda\left(1+\left(\beta / x_{i: m: n}\right)\right)^{-(\alpha+1)}}{x_{i: m: n}\left(1+\lambda-\lambda\left(1+\left(\beta / x_{i: m: n}\right)\right)^{-\alpha}\right)} \\
& +\sum_{i=1}^{m} R_{i}\left[\frac{\alpha \lambda\left(1+\left(\beta / x_{i: m: n}\right)\right)^{-(\alpha+1)}}{x_{i: m: n}\left(1+\lambda-\lambda\left(1+\left(\beta / x_{i: m: n}\right)\right)^{-\alpha}\right)\left(\ln \left(1+\lambda-\lambda\left(1+\left(\beta / x_{i: m: n}\right)\right)^{-\alpha}\right)\right)}\right] \\
\frac{\partial \ell}{\partial \lambda}= & \frac{m}{\lambda}-\frac{m}{(\ln (1+\lambda))(1+\lambda)}-\sum_{i=1}^{m} \frac{1-\left(1+\left(\beta / x_{i: m: n}\right)\right)^{-\alpha}}{\left(1+\lambda-\lambda\left(1+\left(\beta / x_{i: m: n}\right)\right)^{-\alpha}\right)} \\
& +\sum_{i=1}^{m} R_{i}\left[\frac{1-\left(1+\left(\beta / x_{i: m: n}\right)\right)^{-\alpha}}{\left(1+\lambda-\lambda\left(1+\left(\beta / x_{i: m: n}\right)\right)^{-\alpha}\right)\left(\ln \left(1+\lambda-\lambda\left(1+\left(\beta / x_{i: m: n}\right)\right)^{-\alpha}\right)\right)}-\frac{(\ln (1+\lambda))(1+\lambda)}{1}\right]
\end{aligned}
$$

Since equations (7)-(9) do not have closed-form solutions, the Newton-Raphson iteration method is used to obtain the estimates. Ahmed [24] and Mahmoud et al. [25] describe the steps in which the algorithm works in detail.

The algorithm is described as follows:

(1) Use the method of moments or any other methods to estimate the parameters $\alpha, \beta$, and $\lambda$ as starting point of iteration, denote the estimates as $\left(\alpha_{0}, \beta_{0}, \lambda_{0}\right)$, and set $k=0$.

(2) Calculate $(\partial \ell / \partial \alpha, \partial \ell / \partial \beta, \partial \ell / \partial \lambda)_{\left(\alpha_{k}, \beta_{k}, \lambda_{k}\right)}$ and the observed Fisher information matrix $I^{-1}(\alpha, \beta, \lambda)$, given in the next section.

(3) Update $(\alpha, \beta, \lambda)$ as

$$
\begin{aligned}
\left(\alpha_{k+1}, \beta_{k+1}, \lambda_{k+1}\right)= & \left(\alpha_{k}, \beta_{k}, \lambda_{k}\right)+\left(\frac{\partial \ell}{\partial \alpha}, \frac{\partial \ell}{\partial \beta}, \frac{\partial \ell}{\partial \lambda}\right)_{\left(\alpha_{k}, \beta_{k}, \lambda_{k}\right)} \\
& \times I^{-1}(\alpha, \beta, \lambda) .
\end{aligned}
$$

(4) Set $k=k+1$ and then go back to Step 1 .

(5) Continue the iterative steps until $\mid\left(\alpha_{k+1}, \beta_{k+1}, \lambda_{k+1}\right)-$ $\left(\alpha_{k}, \beta_{k}, \lambda_{k}\right) \mid$ is smaller than a threshold value. The final estimates of $(\alpha, \beta, \lambda)$ are the MLE of the parameters denoted as $(\widehat{\alpha}, \widehat{\beta}, \widehat{\lambda})$.
Moreover, the invariance property of MLEs, and the MLEs of $S(t)$ and $h(t)$ can be obtained after replacing $\alpha, \beta$, and $\lambda$ by $\widehat{\alpha}, \widehat{\beta}$, and $\hat{\lambda}$ as

$$
\begin{aligned}
& \widehat{S}(t)=\frac{\log \left[1+\hat{\lambda}-\hat{\lambda}(1+(\widehat{\beta} / t))^{-\widehat{\alpha}}\right]}{\log (1+\widehat{\lambda})}, \\
& \widehat{h}(t)=\frac{\left(\widehat{\alpha} \widehat{\beta} \widehat{\lambda} / t^{2}\right)(1+(\widehat{\beta} / t))^{-(\widehat{\alpha}+1)}}{\left[1+\widehat{\lambda}-\hat{\lambda}(1+(\widehat{\beta} / t))^{-\widehat{\alpha}}\right] \log \left[1+\widehat{\lambda}-\hat{\lambda}(1+(\widehat{\beta} / t))^{-\widehat{\alpha}}\right]} .
\end{aligned}
$$

\section{Asymptotic Confidence Intervals}

The asymptotic variances and covariances of the MLEs, $\widehat{\alpha}, \widehat{\beta}$, and $\hat{\lambda}$ are given by the entries of the inverse of the Fisher information matrix $I_{i j}=E\left\{-\left[\partial^{2} \ell(\Psi) / \partial \psi_{i} \partial \psi_{j}\right]\right\}$, where $i, j=$ $1,2,3$ and $\Psi=\left(\psi_{1}, \psi_{2}, \psi_{3}\right)=(\alpha, \beta, \lambda)$. Unfortunately, exact closed forms for the expectations listed above are difficult to come by. Therefore, the observed Fisher information matrix $\widehat{I}_{i j}=\left\{-\left[\partial^{2} \ell(\Psi) / \partial \psi_{i} \partial \psi_{j}\right]\right\}_{\Psi=\widehat{\Psi}}$, which is obtained by dropping the expectation operator $E$, will be used to construct confidence intervals (CIs) for the parameters. As a result, the observed information matrix is given by 


$$
\begin{aligned}
& \widehat{I}(\widehat{\alpha}, \widehat{\beta}, \widehat{\lambda})=\left(\begin{array}{rrr}
-\frac{\partial^{2} \ell}{\partial \alpha^{2}} & -\frac{\partial^{2} \ell}{\partial \alpha \partial \beta} & -\frac{\partial^{2} \ell}{\partial \alpha \partial \lambda} \\
-\frac{\partial^{2} \ell}{\partial \beta \partial \alpha} & -\frac{\partial^{2} \ell}{\partial \beta^{2}} & -\frac{\partial^{2} \ell}{\partial \beta \partial \lambda} \\
-\frac{\partial^{2} \ell}{\partial \lambda \partial \alpha} & -\frac{\partial^{2} \ell}{\partial \lambda \partial \beta} & -\frac{\partial^{2} \ell}{\partial \lambda^{2}}
\end{array}\right)_{(\alpha, \beta, \lambda)=(\widehat{\alpha}, \widehat{\beta}, \widehat{\lambda})} \\
& \frac{\partial^{2} \ell}{\partial \alpha^{2}}=-\frac{m}{\alpha^{2}}+\sum_{i=1}^{m} \frac{\lambda\left(\ln ^{2}\left(1+\left(\beta / x_{i: m: n}\right)\right)\right)(\lambda+1)\left(1+\left(\beta / x_{i: m: n}\right)\right)^{\alpha}}{\left(\lambda\left(1+\left(\beta / x_{i: m: n}\right)\right)^{\alpha}-\lambda+\left(1+\left(\beta / x_{i: m: n}\right)\right)^{\alpha}\right)^{2}}-\sum_{i=1}^{m} R_{i}\left[\frac{\lambda\left(\ln ^{2}\left(1+\left(\beta / x_{i: m: n}\right)\right)\right)}{\ln ^{2}\left(1+\lambda-\lambda\left(1+\left(\beta / x_{i: m: n}\right)\right)^{-\alpha}\right)}\right. \\
& \left.\times \frac{\lambda+\left(\ln \left(1+\lambda-\lambda\left(1+\left(\beta / x_{i: m: n}\right)\right)^{-\alpha}\right)\left(1+\left(\beta / x_{i: m: n}\right)\right)^{\alpha}\right)(1+\lambda)}{\left(\lambda\left(1+\left(\beta / x_{i: m: n}\right)\right)^{\alpha}-\lambda+\left(1+\left(\beta / x_{i: m: n}\right)\right)^{\alpha}\right)^{2}}\right] \text {, } \\
& \frac{\partial^{2} \ell}{\partial \beta^{2}}=-\frac{m}{\beta^{2}}+\sum_{i=1}^{m} \frac{\alpha+1}{\left(\beta+x_{i: m: n}\right)^{2}}+\sum_{i=1}^{m}\left[\frac{\alpha \lambda(\alpha+1)\left(1+\lambda-\lambda\left(1+\left(\beta / x_{i: m: n}\right)\right)^{-\alpha}\right)}{x^{2}\left(1+\lambda-\lambda\left(1+\left(\beta / x_{i: m: n}\right)\right)^{-\alpha}\right)^{2}} \times\left(1+\frac{\beta}{x_{i: m: n}}\right)^{-(\alpha+2)}-\alpha^{2} \lambda^{2}\left(1+\frac{\beta}{x_{i: m: n}}\right)^{-(2 \alpha+2)}\right] \\
& -\sum_{i=1}^{m} R_{i}\left[\frac{\alpha \lambda}{x^{2}\left(\ln ^{2}\left(1+\lambda-\lambda\left(1+\left(\beta / x_{i: m: n}\right)\right)^{-\alpha}\right)\right)\left(1+\lambda-\lambda\left(1+\left(\beta / x_{i: m: n}\right)\right)^{-\alpha}\right)^{2}}\right. \\
& \left.\times\left(\frac{\ln \left(1+\lambda-\lambda\left(1+\left(\beta / x_{i: m: n}\right)\right)^{-\alpha}\right)(1+\alpha+\lambda+\alpha \lambda)}{\left(1+\left(\beta / x_{i: m: n}\right)\right)^{\alpha+2}}-\frac{\lambda \ln \left(1+\lambda-\lambda\left(1+\left(\beta / x_{i: m: n}\right)\right)^{-\alpha}\right)+\alpha \lambda}{\left(1+\left(\beta / x_{i: m: n}\right)\right)^{2 \alpha+2}}\right)\right], \\
& \frac{\partial^{2} \ell}{\partial \lambda^{2}}=-\frac{m}{\lambda^{2}}+\frac{m(\ln (\lambda+1)+1)}{\left(\ln ^{2}(\lambda+1)\right)(\lambda+1)^{2}}+\sum_{i=1}^{m} \frac{\left(\left(1+\left(\beta / x_{i: m: n}\right)\right)^{-\alpha}-1\right)^{2}}{\left(1+\lambda-\lambda\left(1+\left(\beta / x_{i: m: n}\right)\right)^{-\alpha}\right)^{2}} \\
& -\sum_{i=1}^{m} R_{i}\left[\frac{\left(\left(1+\left(\beta / x_{i: m: n}\right)\right)^{-\alpha}-1\right)^{2}\left(\ln \left(1+\lambda-\lambda\left(1+\left(\beta / x_{i: m: n}\right)\right)^{-\alpha}\right)+1\right)}{\left(1+\lambda-\lambda\left(1+\left(\beta / x_{i: m: n}\right)\right)^{-\alpha}\right)^{2} \ln ^{2}\left(1+\lambda-\lambda\left(1+\left(\beta / x_{i: m: n}\right)\right)^{-\alpha}\right)}-\frac{(\ln (\lambda+1)+1)}{\left(\ln ^{2}(\lambda+1)\right)(\lambda+1)^{2}}\right] \text {, }
\end{aligned}
$$

$$
\begin{aligned}
\frac{\partial^{2} \ell}{\partial \alpha \partial \beta}= & -\sum_{i=1}^{m} \frac{1}{x_{i: m: n}+\beta}+\sum_{i=1}^{m}\left[\frac{\lambda}{\left(1+\left(\beta / x_{i: m: n}\right)\right)^{2 \alpha}\left(x_{i: m: n}+\beta\right)\left(1+\lambda-\lambda\left(1+\left(\beta / x_{i: m: n}\right)\right)^{-\alpha}\right)^{2}}\right. \\
& \times \lambda-\lambda\left(1+\frac{\beta}{x_{i: m: n}}\right)^{\alpha}-\left(1+\frac{\beta}{x_{i: m: n}}\right)^{\alpha}+\alpha\left(\left(\ln \left(1+\frac{\beta}{x_{i: m: n}}\right)\right)\left(1+\frac{\beta}{x_{i: m: n}}\right)^{\alpha}+\alpha \lambda\left(\ln \left(1+\frac{\beta}{x_{i: m: n}}\right)\right)\left(1+\frac{\beta}{x_{i: m: n}}\right)^{\alpha}\right) \\
& -\sum_{i=1}^{m} R_{i}\left[\frac{\lambda\left(1+\lambda-\lambda\left(1+\left(\beta / x_{i: m: n}\right)\right)^{-\alpha}\right)^{-2}\left(1+\left(\beta / x_{i: m: n}\right)\right)^{-2 \alpha}}{\left(\ln ^{2}\left(1+\lambda-\lambda\left(1+\left(\beta / x_{i: m: n}\right)\right)^{-\alpha}\right)\right)\left(x_{i: m: n}+\beta\right)}\right. \\
& +\left(\lambda \ln \left(1+\lambda-\lambda\left(1+\frac{\beta}{x_{i: m: n}}\right)^{-\alpha}\right)-\left(\ln \left(1+\lambda-\lambda\left(1+\frac{\beta}{x_{i: m: n}}\right)^{-\alpha}\right)\right)\left(1+\frac{\beta}{x_{i: m: n}}\right)^{\alpha}\right. \\
& -\lambda\left(\ln \left(1+\lambda-\lambda\left(1+\frac{\beta}{x_{i: m: n}}\right)^{-\alpha}\right)\right)\left(1+\frac{\beta}{x_{i: m: n}}\right)^{\alpha}+\alpha \lambda \ln \left(1+\frac{\beta}{x_{i: m: n}}\right) \\
& +\alpha\left(\ln \left(1+\frac{\beta}{x_{i: m: n}}\right) \ln \left(1+\lambda-\lambda\left(1+\frac{\beta}{x_{i: m: n}}\right)^{-\alpha}\right)\right)\left(1+\frac{\beta}{x_{i: m: n}}\right)^{\alpha} \\
& \left.\left.+\alpha \lambda\left(\ln \left(1+\frac{\beta}{x_{i: m: n}}\right) \ln \left(1+\lambda-\lambda\left(1+\frac{\beta}{x_{i: m: n}}\right)^{-\alpha}\right)\right)\left(1+\frac{\beta}{x_{i: m: n}}\right)^{\alpha}\right)\right]
\end{aligned}
$$




$$
\begin{aligned}
\frac{\partial^{2} \ell}{\partial \alpha \partial \lambda}= & -\sum_{i=1}^{m} \frac{\left(1+\left(\beta / x_{i: m: n}\right)\right)^{-\alpha} \ln \left(1+\left(\beta / x_{i: m: n}\right)\right)}{\left(1+\lambda-\lambda\left(1+\left(\beta / x_{i: m: n}\right)\right)^{-\alpha}\right)^{2}}+\sum_{i=1}^{m} R_{i}\left[\frac{\ln \left(1+\left(\beta / x_{i: m: n}\right)\right)}{\left(\ln ^{2}\left(1+\lambda-\lambda\left(1+\left(\beta / x_{i: m: n}\right)\right)^{-\alpha}\right)\right)}\right. \\
& \left.\times \frac{\left(\ln \left(1+\lambda-\lambda\left(1+\left(\beta / x_{i: m: n}\right)\right)^{-\alpha}\right) \lambda-\lambda\left(1+\left(\beta / x_{i: m: n}\right)\right)^{-\alpha}\right)}{\left(1+\left(\beta / x_{i: m: n}\right)\right)^{\alpha}\left(1+\lambda-\lambda\left(1+\left(\beta / x_{i: m: n}\right)\right)^{-\alpha}\right)^{2}}\right], \\
\frac{\partial^{2} \ell}{\partial \beta \partial \lambda}= & -\sum_{i=1}^{m} \frac{\alpha\left(1+\left(\beta / x_{i: m: n}\right)\right)^{-(\alpha+1)}}{x_{i: m: n}\left(1+\lambda-\lambda\left(1+\left(\beta / x_{i: m: n}\right)\right)^{-\alpha}\right)^{2}} \\
& +\sum_{i=1}^{m} R_{i}\left[\frac{\alpha\left(\ln \left(1+\lambda-\lambda\left(1+\left(\beta / x_{i: m: n}\right)\right)^{-\alpha}\right) \lambda-\lambda\left(1+\left(\beta / x_{i: m: n}\right)\right)^{-\alpha}\right)\left(1+\left(\beta / x_{i: m: n}\right)\right)^{-(\alpha+1)}}{x_{i: m: n}\left(\ln ^{2}\left(1+\lambda-\lambda\left(1+\left(\beta / x_{i: m: n}\right)\right)^{-\alpha}\right)\right)\left(1+\lambda-\lambda\left(1+\left(\beta / x_{i: m: n}\right)\right)^{-\alpha}\right)^{2}}\right] .
\end{aligned}
$$

Therefore, the approximate asymptotic variance-covariance matrix $[\widehat{V}]$ for the MLEs is obtained by inverting the observed information matrix $\widehat{I}(\alpha, \beta, \lambda)$; therefore,

$$
[\widehat{V}]=\widehat{I}^{-1}(\widehat{\alpha}, \widehat{\beta}, \widehat{\lambda})=\left(\begin{array}{ccc}
\operatorname{Var}(\widehat{\alpha}) & \operatorname{cov}(\widehat{\alpha}, \widehat{\beta}) & \operatorname{cov}(\widehat{\alpha}, \widehat{\lambda}) \\
\operatorname{cov}(\widehat{\beta}, \widehat{\alpha}) & \operatorname{Var}(\widehat{\beta}) & \operatorname{cov}(\widehat{\beta}, \widehat{\lambda}) \\
\operatorname{cov}(\widehat{\lambda}, \widehat{\alpha}) & \operatorname{cov}(\widehat{\lambda}, \widehat{\beta}) & \operatorname{Var}(\widehat{\lambda})
\end{array}\right)
$$

It is well known that under some regularity conditions (see Lawless [26]), ( $\widehat{\alpha}, \widehat{\beta}, \widehat{\lambda})$ is approximately distributed as multivariate normal with mean $(\alpha, \beta, \lambda)$ and covariance matrix $I^{-1}(\alpha, \beta, \lambda)$. Then, the $100(1-\gamma) \%$ two-sided confidence intervals of $\alpha, \beta$, and $\lambda$ can be given by

$$
\begin{aligned}
& \left(\widehat{\alpha} \pm Z_{(\gamma / 2)} \sqrt{\operatorname{var}(\widehat{\alpha})}\right), \\
& \left(\widehat{\beta} \pm Z_{(\gamma / 2)} \sqrt{\operatorname{var}(\widehat{\beta})}\right), \\
& \left(\hat{\lambda} \pm Z_{(\gamma / 2)} \sqrt{\operatorname{var}(\widehat{\lambda})}\right),
\end{aligned}
$$

where $Z_{(\gamma / 2)}$ is the percentile of the standard normal distribution with right-tail probability $(\gamma / 2)$.

To construct the asymptotic confidence interval of the reliability and hazard functions, which are functions in the parameters $\alpha, \beta$, and $\lambda$, it is necessary to compute their variances. The delta method is used to compute the approximate estimates of the variance of $\widehat{S}(t)$ and $\widehat{h}(t)$ (for more details about the delta method, see Greene [27], Ahmed [24], and El-Sagheer [28]). According to this method, the variance of $\widehat{S}(t)$ and $\widehat{h}(t)$ can be approximated, respectively, by

$$
\begin{gathered}
\widehat{\sigma}_{\widehat{S}(t)}^{2}=[\nabla \widehat{S}(t)]^{T}[\widehat{V}][\nabla \widehat{S}(t)], \\
\widehat{\sigma}_{\widehat{h}(t)}^{2}=[\nabla \widehat{h}(t)]^{T}[\widehat{V}][\nabla \widehat{h}(t)],
\end{gathered}
$$

where $\nabla \widehat{S}(t)$ and $\nabla \widehat{h}(t)$ are, respectively, the gradient of $\widehat{S}(t)$ and $\widehat{h}(t)$ with respect to $\alpha, \beta$, and $\lambda$.

Then, the $100(1-\gamma) \%$ two-sided confidence intervals of $S(t)$ and $h(t)$ can be given by

$$
\begin{aligned}
& \widehat{S}(t) \pm Z_{(\gamma / 2)} \sqrt{\widehat{\sigma}_{\widehat{S}(t)}^{2}}, \\
& \widehat{h}(t) \pm Z_{(\gamma / 2)} \sqrt{\widehat{\sigma}_{\widehat{h}(t)}^{2}}
\end{aligned}
$$

\section{Bootstrap Confidence Intervals}

A parametric bootstrap interval, as opposed to a point estimate, substantially provides more information about the population value of the quantity of interest. In addition, it is clear that confidence intervals based on asymptotic results perform poorly for a small sample size. Two parametric bootstrap algorithms are offered to calculate the bootstrap confidence intervals of $\alpha, \beta, \lambda, S(t)$ and $h(t)$. The first one is the percentile bootstrap (Boot-p) confidence interval, proposed by Efron [29]. The second one is the bootstrap-t (Boott) confidence interval, which is presented in Hall [30]. Boot- $t$ was created using a studentized "pivot" and requires an estimator of the variance of the MLE of $\alpha, \beta, \lambda, S(t)$ and $h(t)$.

\subsection{Parametric Boot- $p$}

(1) Based on the original data $x=x_{1: m: n}, x_{2: m: n}, \ldots$, $x_{m: m: n}, \widehat{\alpha}, \widehat{\beta}$, and $\widehat{\lambda}$ are obtain by maximizing equations (7)-(9).

(2) Based on the prespecified progressive censoring scheme $\left(R_{1}, R_{2}, \ldots, R_{m}\right)$, a type-II progressive censoring sample $x^{*}=x_{1: m: n}^{*}, x_{2: m: n}^{*}, \ldots, x_{m: m: n}^{*}$ from the FRL-IL distribution with parameters $\widehat{\alpha}, \hat{\beta}$, and $\widehat{\lambda}$ are generated, using the algorithm described in Balakrishnan and Sandhu [11].

(3) The MLEs are obtained based on the bootstrap sample, and this bootstrap estimate is denoted by $\widehat{\psi}^{*}$ (in our case $\psi$ could) be $\alpha, \beta, \lambda, S(t)$ and $h(t)$.

(4) Steps (2) and (3) are repeated $N$ boot times, and $\widehat{\psi}_{1}^{*}, \widehat{\psi}_{*}^{*}, \ldots, \widehat{\psi}_{N_{*} \text { boot }}^{*}$ is obtained, where $\widehat{\psi}_{i}^{*}=\left(\widehat{\alpha}_{i}^{*}\right.$, $\left.\hat{\beta}_{i}^{*}, \hat{\lambda}_{i}^{*}, \widehat{S}_{i}^{*}(t), \hat{h}_{i}^{*}(t)\right), i=1,2,3, \ldots, N$ boot.

(5) $\widehat{\psi}_{i}^{*}, i=1,2,3, \ldots, N$ boot is arranged in ascending orders, and $\widehat{\psi}_{(1)}^{*}, \widehat{\psi}_{(2)}^{*}, \ldots, \widehat{\psi}_{(N \text { boot })}^{*}$ is obtained. 
Let $G_{1}(z)=P\left(\widehat{\psi}^{*} \leq z\right)$ be the cumulative distribution function of $\widehat{\psi}^{*} . \widehat{\psi}_{\text {boot }-p}=G_{1}^{-1}(z)$ is defined for given $z$. The approximate bootstrap-p $100(1-\gamma) \%$ CI of $\widehat{\psi}$ is given by

$$
\left[\widehat{\psi}_{\text {boot }-p}\left(\frac{\gamma}{2}\right), \widehat{\psi}_{\text {boot }-p}\left(1-\frac{\gamma}{2}\right)\right] \text {. }
$$

\subsection{Parametric Boot-t}

(1)-(3) The same as the parametric Boot-p.

(4) Based on the asymptotic variance-covariance matrix (19) and delta method (21), respectively, the variancecovariance matrix $I^{-1^{*}}(\partial \ell / \partial \alpha, \partial \ell / \partial \beta, \partial \ell / \partial \lambda)$ and the approximate estimates of the variance $S(t)$ and $h(t)$ are computed.

(5) The $T^{* \psi}$ statistic is computed, which is defined as

$$
T^{* \psi}=\frac{\left(\widehat{\psi}^{*}-\widehat{\psi}\right)}{\sqrt{\operatorname{var}\left(\widehat{\psi}^{*}\right)}} .
$$

(6) Steps 2-5 are repeated NBoot times, and $T_{1}^{* \psi}, T_{2}^{* \psi}, \ldots, T_{N \text { boot }}^{* \psi}$ is obtained.

(7) $T_{1}^{* \psi}, T_{2}^{* \psi}, \ldots, T_{N \text { boot }}^{* \psi}$ is sorted in ascending orders, and the ordered sequences $T_{(1)}^{* \psi}, T_{(2)}^{* \psi}, \ldots, T_{(N \text { boot })}^{* \psi}$ are obtained.

Let $G_{2}(z)=P\left(T^{*} \leq z\right)$ be the cumulative distribution function of $T^{*}$ for given $z \cdot \widehat{\psi}_{\text {boot-t }}=\widehat{\psi}+G_{2}^{-1}(z) \sqrt{\operatorname{var(\widehat {\psi }^{*})}}$ is defined.

Then, the approximate bootstrap-t $100(1-\gamma) \%$ CI of $\widehat{\psi}=(\widehat{\alpha}, \widehat{\beta}, \widehat{\lambda}, \widehat{S}(t), \widehat{h}(t))$ is given by

$$
\left[\widehat{\psi}_{\text {boot }-t}\left(\frac{\gamma}{2}\right), \widehat{\psi}_{\text {boot }-p}\left(1-\frac{\gamma}{2}\right)\right] .
$$

\section{Bayes Estimation}

In this section, we obtain Bayesian estimates that deal with the parameters as random and uncertainties in the parameters are described by a joint prior distribution, which was developed before the collected failure data. The Bayesian approach is highly useful in reliability analysis because it may incorporate previous knowledge into the analysis. This is important because one of the main challenges associated with reliability analysis is the limited availability of data. Bayesian estimates of the unknown parameters $\alpha, \beta$, and $\lambda$ and some lifetime parameters $S(t)$ and $h(t)$ against the SEL and LINEX loss functions are developed. It is assumed here that the parameters $\alpha, \beta$, and $\lambda$ are independent and follow the gamma prior distributions,

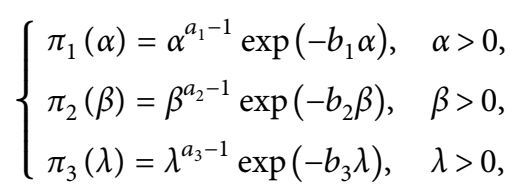

where all the hyperparameters $a_{i}$ and $b_{i}, i=1,2,3$ are assumed to be non-negative and known.

The posterior distribution of the parameters $\alpha, \beta$, and $\lambda$ denoted by $\pi^{*}(\alpha, \beta, \lambda \mid \underline{x})$ can be obtained by combining the likelihood function in equation (4) with the priors in equation (23), and it can be written as

$\pi^{*}(\alpha, \beta, \lambda \mid \underline{x})=\frac{\pi_{1}(\alpha) \pi_{2}(\beta) \pi_{3}(\lambda) L(\alpha, \beta, \lambda \mid \underline{x})}{\int_{0}^{\infty} \int_{0}^{\infty} \int_{0}^{\infty} \pi_{1}(\alpha) \pi_{2}(\beta) \pi_{3}(\lambda) L(\alpha, \beta, \lambda \mid \underline{x}) \mathrm{d} \alpha \mathrm{d} \beta \mathrm{d} \lambda}$.

A commonly used loss function is the SEL, which is a symmetrical loss function that assigns equal losses to overestimation and underestimation. If $\phi$ is the parameter to be estimated by an estimator $\hat{\phi}$, then the square error loss function is defined as

$$
L(\phi, \widehat{\phi})=(\widehat{\phi}-\phi)^{2} .
$$

Therefore, the Bayes estimate of any function of $\alpha, \beta$, and $\lambda$, say $g(\alpha, \beta, \lambda)$ under the SEL function, can be obtained as

$$
\widehat{g}_{\mathrm{BS}}(\alpha, \beta, \lambda \mid \underline{x})=E_{\alpha, \beta, \lambda \mid \underline{x}}(g(\alpha, \beta, \lambda)),
$$

where

$$
E_{\alpha, \beta, \lambda \mid \underline{x}}(g(\alpha, \beta, \lambda))=\frac{\int_{0}^{\infty} \int_{0}^{\infty} \int_{0}^{\infty} g(\alpha, \beta, \lambda) \pi_{1}(\alpha) \pi_{2}(\beta) \pi_{3}(\lambda) L(\alpha, \beta, \lambda \mid \underline{x}) \mathrm{d} \alpha \mathrm{d} \beta \mathrm{d} \lambda}{\int_{0}^{\infty} \int_{0}^{\infty} \int_{0}^{\infty} \pi_{1}(\alpha) \pi_{2}(\beta) \pi_{3}(\lambda) L(\alpha, \beta, \lambda \mid \underline{x}) \mathrm{d} \alpha \mathrm{d} \beta \mathrm{d} \lambda}
$$

Variance [31] considered a LINEX (linear exponential) loss function $L(\Delta)$ for a parameter $\phi$ is given by

$$
L(\Delta)=\left(e^{c \Delta}-c \Delta-1\right), \quad c \neq 0, \Delta=\widehat{\phi}-\phi .
$$

This loss function is suitable for situations where overestimation is more costly than its underestimation. Zellner [32] discussed Bayesian estimation and prediction using LINEX loss. Hence, under LINEX loss function in equation (30), the Bayes estimate of a function $g(\alpha, \beta, \lambda)$ is

$$
\widehat{g}_{\mathrm{BL}}(\alpha, \beta, \lambda \mid \underline{x})=-\frac{1}{c} \log \left[E\left(e^{-c g(\alpha, \beta, \lambda)} \mid \underline{x}\right)\right], \quad c \neq 0,
$$

where 


$$
E\left(e^{-c g(\alpha, \beta, \lambda)} \mid \underline{x}\right)=\frac{\int_{0}^{\infty} \int_{0}^{\infty} \int_{0}^{\infty} e^{-c g(\alpha, \beta, \lambda)} \pi_{1}(\alpha) \pi_{2}(\beta) \pi_{3}(\lambda) L(\alpha, \beta, \lambda \mid \underline{x}) \mathrm{d} \alpha \mathrm{d} \beta \mathrm{d} \lambda}{\int_{0}^{\infty} \int_{0}^{\infty} \int_{0}^{\infty} \pi_{1}(\alpha) \pi_{2}(\beta) \pi_{3}(\lambda) L(\alpha, \beta, \lambda \mid \underline{x}) \mathrm{d} \alpha \mathrm{d} \beta \mathrm{d} \lambda},
$$

where the calculation of the multiple integrals in equations (30) and (33) cannot be analytically solved. Thus, the MCMC technique is used to generate samples from the joint posterior density function in equation (27). In order to be able to implement the MCMC technique, we consider the Gibbs within the Metropolis-Hasting sampler procedure. The $\mathrm{M}-\mathrm{H}$ and Gibbs sampling are two useful MCMC methods that have been widely used in statistics. These methods recently have a high degree of discussions. (for details, see Ahmed [33], Panahi and Asadi [34], and Abushal [35]).

From equation (27), the joint posterior distribution can be written as follows:

$$
\begin{aligned}
\pi^{*}(\alpha, \beta, \lambda \mid \underline{x}) & \propto \frac{\alpha^{m+a_{1}-1} \beta^{m+a_{2}-1} \lambda^{m+a_{3}-1} e^{-b_{1} \alpha-b_{2} \beta-b_{3} \lambda} \prod_{i=1}^{m} x_{i: m: n}^{-2} \prod_{i=1}^{m}\left(1+\left(\beta / x_{i: m: n}\right)\right)^{-(\alpha+1)}}{\prod_{i=1}^{m}\left(1+\lambda-\lambda\left(1+\left(\beta / x_{i: m: n}\right)\right)^{-\alpha}\right)(\log (1+\lambda))^{m}} \\
& \times \prod_{i=1}^{m}\left(\frac{\log \left[1+\lambda-\lambda\left(1+\left(\beta / x_{i: m: n}\right)\right)^{-\alpha}\right]}{\log [1+\lambda]}\right)^{R_{i}} .
\end{aligned}
$$

The posterior conditionals for $\alpha, \beta$, and $\lambda$ are

$$
\begin{aligned}
& \pi_{1}^{*}(\alpha \mid \beta, \lambda, \underline{x}) \propto \alpha^{m+a_{1}-1} e^{-b_{1} \alpha} \prod_{i=1}^{m} x_{i: m: n}^{-2} \frac{\prod_{i=1}^{m}\left(1+\left(\beta / x_{i: m: n}\right)\right)^{-(\alpha+1)}}{\prod_{i=1}^{m}\left(1+\lambda-\lambda\left(1+\left(\beta / x_{i: m: n}\right)\right)^{-\alpha}\right) \times \prod_{i=1}^{m}\left(\frac{\log \left[1+\lambda-\lambda\left(1+\left(\beta / x_{i: m: n}\right)\right)^{-\alpha}\right]}{\log [1+\lambda]}\right)^{R_{i}}}, \\
& \pi_{2}^{*}(\beta \mid \alpha, \lambda, \underline{x}) \propto \beta^{m+a_{2}-1} e^{-b_{2} \beta} \prod_{i=1}^{m} x_{i: m: n}^{-2} \frac{\prod_{i=1}^{m}\left(1+\left(\beta / x_{i: m: n}\right)\right)^{-(\alpha+1)}}{\prod_{i=1}^{m}\left(1+\lambda-\lambda\left(1+\left(\beta / x_{i: m: n}\right)\right)^{-\alpha}\right) \times \prod_{i=1}^{m}\left(\frac{\log \left[1+\lambda-\lambda\left(1+\left(\beta / x_{i: m: n}\right)\right)^{-\alpha}\right]}{\log [1+\lambda]}\right)^{R_{i}}}, \\
& \pi_{3}^{*}(\lambda \mid \alpha, \beta, \underline{x}) \propto \lambda^{m+a_{3}-1} e^{-b_{3} \lambda} \prod_{i=1}^{m} x_{i: m: n}^{-2} \frac{\prod_{i=1}^{m}\left(1+\left(\beta / x_{i: m: n}\right)\right)^{-(\alpha+1)}}{\prod_{i=1}^{m}\left(1+\lambda-\lambda\left(1+\left(\beta / x_{i: m: n}\right)\right)^{-\alpha}\right)(\log (1+\lambda))^{m} \times \prod_{i=1}^{m}\left(\frac{\log \left[1+\lambda-\lambda\left(1+\left(\beta / x_{i: m: n}\right)\right)^{-\alpha}\right]}{\log [1+\lambda]}\right)^{R_{i}}} .
\end{aligned}
$$

But, the conditional posteriors of $\alpha, \beta$, and $\lambda$ in equations (35)-(37) do not present standard forms, so Gibbs sampling is not a straightforward option. Therefore, the use of the Metropolis-Hasting sampler is required for the implementation of MCMC technique. Within Gibbs sampling, the Metropolis-Hasting algorithm is as follows:

(1) Start with an initial guess $\left(\alpha^{(0)}, \beta^{(0)}, \lambda^{(0)}\right)$.

(2) Set $j=1$.

(3) Using the following M-H algorithm, generate $\alpha^{(j)}, \beta^{(j)}$, and $\lambda^{(j)}$ from $\pi_{1}^{*}\left(\alpha^{(j-1)} \mid \beta^{(j-1)}, \lambda^{(j-1)}, \underline{x}\right), \quad \pi_{2}^{*}$ $\left(\beta^{(j-1)} \mid \alpha^{(j)}, \lambda^{(j-1)}, \underline{x}\right)$ and $\pi_{3}^{*}\left(\lambda^{(j-1)} \mid \alpha^{(j)}, \beta^{(j)}, \underline{x}\right)$ with the normal proposal distributions

$$
\begin{aligned}
& N\left(\alpha^{(j-1)}, \operatorname{var}(\alpha)\right), \\
& N\left(\beta^{(j-1)}, \operatorname{var}(\beta)\right), \\
& N\left(\lambda^{(j-1)}, \operatorname{var}(\lambda)\right),
\end{aligned}
$$

where $\operatorname{var}(\alpha), \operatorname{var}(\beta)$, and $\operatorname{var}(\lambda)$ can be obtained from the main diagonal in inverse Fisher information matrix in equation (12).

(4) Generate a proposal $\alpha^{*}$ from $N\left(\alpha^{(j-1)}, \operatorname{var}(\alpha)\right), \beta^{*}$ from $N\left(\beta^{(j-1)}, \operatorname{var}(\alpha)\right), \quad$ and $\lambda^{*}$ from $N\left(\lambda^{(j-1)}, \operatorname{var}(\lambda)\right)$.

(i) Evaluate the acceptance probabilities 


$$
\left.\begin{array}{l}
\eta_{\alpha}=\min \left[1, \frac{\pi_{1}^{*}\left(\alpha^{*} \mid \beta^{(j-1)}, \lambda^{(j-1)}, \underline{x}\right)}{\pi_{1}^{*}\left(\alpha^{(j-1)} \mid \beta^{(j-1)}, \lambda^{(j-1)}, \underline{x}\right)}\right] \\
\left.\eta_{\beta}=\min \left[1, \frac{\pi_{2}^{*}\left(\beta^{*} \mid \alpha^{(j)}, \lambda^{(j-1)}, \underline{x}\right)}{\pi_{2}^{*}\left(\beta^{(j-1)} \mid \alpha^{(j)}, \lambda^{(j-1)}, \underline{x}\right)}\right]\right\} . \\
\eta_{\lambda}=\min \left[1, \frac{\pi_{3}^{*}\left(\lambda^{*} \mid \alpha^{(j)}, \beta^{(j)}, \underline{x}\right)}{\pi_{3}^{*}\left(\lambda^{(j-1)} \mid \alpha^{(j)}, \beta^{(j)}, \underline{x}\right)}\right]
\end{array}\right\}
$$

$$
\begin{cases}S^{(j)}(t)=\frac{\log \left[1+\lambda^{(j)}-\lambda^{(j)}\left(1+\left(\beta^{(j)} / t\right)\right)^{\alpha^{(j)}}\right]}{\log \left(1+\lambda^{(j)}\right)}, & t \geq 0, \\ h^{(j)}(t)=\frac{\left(\alpha^{(j)} \beta^{(j)} \lambda^{(j)} / t^{2}\right)\left(1+\left(\beta^{(j)} / t\right)\right)^{-\left(\alpha^{(j)}+1\right)}}{\left[1+\lambda^{(j)}-\lambda^{(j)}\left(1+\left(\beta^{(j)} / t\right)\right)^{\alpha^{(j)}}\right] \log \left[1+\lambda^{(j)}-\lambda^{(j)}\left(1+\left(\beta^{(j)} / t\right)\right)^{\alpha^{(j)}}\right]}, & t \geq 0 .\end{cases}
$$

(ii) Generate a $u_{1}, u_{2}$, and $u_{3}$ from a uniform $(0,1)$ distribution.

(iii) If $u_{1}<\eta_{\alpha}$, accept the proposal and set $\alpha^{(j)}=\alpha^{*}$, else set $\alpha^{(j)}=\alpha^{(j-1)}$.

(iv) If $u_{2}<\eta_{\beta}$, accept the proposal and set $\beta^{(j)}=\beta^{*}$, else set $\beta^{(j)}=\beta^{(j-1)}$.

(v) If $u_{3}<\eta_{\lambda}$, accept the proposal and set $\lambda^{(j)}=\lambda^{*}$, else set $\lambda^{(j)}=\lambda^{(j-1)}$.

(5) Compute the reliability function and hazard function as
(6) Set $j=j+1$.

(7) Repeat steps (3)-(6) $N$ times and obtain $\alpha^{(i)}, \beta^{(i)}, \lambda^{(i)}, S^{(i)}(t)$ and $h^{(i)}(t), i=1,2, \ldots, N$.

(8) To compute the CRs of $\alpha, \beta, \lambda, S(t)$ and $h(t) \psi_{k}^{(i)}, k=$ $1,2,3,4,5,\left(\psi_{1}, \psi_{2}, \psi_{3}, \psi_{4}, \psi_{5}\right)=(\alpha, \beta, \lambda, S(t), h(t))$ as $\psi_{k}^{(1)}<\psi_{k}^{(2)} \cdots<\psi_{k}^{(N)}$, then the $100(1-\gamma) \%$ CRIs of $\psi_{k}$ is

$$
\left(\psi_{k(N \gamma / 2)}, \psi_{k(N(1-\gamma / 2))}\right) .
$$

The first $M$ simulated variants are discarded in order to ensure convergence, and the affection of initial value selection is removed. Then, the selected samples are $\psi_{k}^{(i)}, j=M+1, \ldots, N$, for sufficiently large $N$.

Based on SEL function, the approximate Bayes estimates of $\psi_{k}$ are given by

$$
\widehat{\psi}_{k}=\frac{1}{N-M} \sum_{j=M+1}^{N} \psi^{(j)} .
$$

The approximate Bayes estimates for $\psi_{k}$, under LINEX loss function, from equation (33), are

$$
\widehat{\psi}_{k}=\frac{-1}{c} \log \left[\frac{1}{N-M} \sum_{j=M+1}^{N} e^{-c \varphi^{(j)}}\right], \quad k=1,2,3,4,5 .
$$

\section{Applications}

In this section, the proposed estimation methods are applied to the failure data for a group of 30 patients with bladder cancer. Bladder cancer is where a growth of abnormal tissue, known as a tumor, develops in the bladder lining. The tumor may migrate into the bladder muscle in some cases (see Lee and Wang [36]). The Kolmogorov-Smirnov (K-S) distance between the empirical distribution of failure data and the CDF of the FRL-IL distribution is 0.111778 with $P$-value that equals 0.0755062 . Hence, the FRL-IL distribution fits well to the given data. A progressive type-II censored sample of effective size $m=15$ was randomly selected from 30 failure observations with a progressive censored scheme $R=(8,7,0,0,0,0,0,0,0,0,0,0,0,0,0)$.

The MLEs of parameters, reliability, and hazard functions based on progressive type-II data presented in Table 1 are obtained to be $\widehat{\alpha}, \widehat{\beta}, \widehat{\lambda}, \widehat{S}(t)$ and $\widehat{h}(t)$ at $t=0.4$ and are displayed in Table 2. Using the algorithms described in Section 4 of the bootstrap methods, the mean of 1000 Boot-p (Bp) and Boot-t (Bt) samples of the lifetime parameters is also displayed in Table 2. The Bayes estimates relative to both SEL and LINEX functions are computed, for different values of the shape parameter $c$ of LINEX loss function for the parameters $\alpha, \beta$, and $\lambda$ and the reliability and hazard functions at $t=0.4$, and are also displayed in Table 2.

The 95\% ACIs and CRIs for the parameters $\alpha, \beta$, and $\lambda$ are computed. Moreover, the delta method is used to obtain the 95\% ACIs and CRIs for the reliability and hazard functions, and the results are displayed in Table 3. Also, the 95\% bootstrap (Boot-p and Boot-t) confidence intervals (CIs) are displayed in Table 3.

It is known that the LINEX loss function becomes symmetric for $c$ tending to zero. From Table 3, the results of SEL and LINEX loss functions, at $c=0.0001$, are the same, indicating that the recommended approaches are accurate. 
TABle 1: Progressive type-II failure data.

\begin{tabular}{lcccrr}
\hline 0.08 & 2.09 & 3.48 & 4.87 & 6.94 & 8.62 \\
13.11 & 23.63 & 0.20 & 2.23 & 7.09 & 4.98 \\
6.97 & 2.26 & 3.57 & 5.06 & 6.64 & 9.22 \\
13.80 & 25.74 & 0.50 & 2.46 & 5.17 & 7.09 \\
7.26 & 9.47 & 2.54 & 3.70 & 7.28 \\
\hline
\end{tabular}

TABle 2: Point estimates for the parameters, reliability, and hazard functions.

\begin{tabular}{|c|c|c|c|c|c|c|c|}
\hline \multirow{3}{*}{ Parameters } & \multirow{3}{*}{ MLE } & \multirow{3}{*}{ Boot-p } & \multirow{3}{*}{ Boot-t } & \multirow{3}{*}{ SEL } & \multirow{2}{*}{\multicolumn{3}{|c|}{$\begin{array}{c}\text { Bayesian } \\
\text { LINEX }\end{array}$}} \\
\hline & & & & & & & \\
\hline & & & & & $c=-2$ & $c=0.0001$ & $c=2$ \\
\hline$\alpha$ & 1.02846 & 1.3246 & 1.20124 & 1.04334 & 1.04429 & 1.04239 & 1.04334 \\
\hline$\beta$ & 1.87912 & 2.5121 & 2.0113 & 2.26516 & 2.29264 & 2.23607 & 2.26516 \\
\hline$\lambda$ & 2.05285 & 2.2451 & 2.0121 & 2.03647 & 2.03791 & 2.03496 & 2.03647 \\
\hline$S$ & 0.869866 & 0.9781 & 0.7584 & 0.891858 & 0.891913 & 0.891804 & 0.891858 \\
\hline$h$ & 0.261585 & 0.34252 & 0.20114 & 0.219842 & 0.220028 & 0.219659 & 0.219842 \\
\hline
\end{tabular}

TABle 3: $95 \%$ CIs of $\alpha, \beta, \lambda, S(t)$ and $h(t)$.

\begin{tabular}{lcccc}
\hline Parameter & MLE & Boot-p & Boot-t & MCMC \\
\hline$\alpha$ & $(-0.104132,2.16105)$ & $(1.24132,2.56203)$ & $(1.1132,1.86203)$ & $(0.987764,1.10752)$ \\
$\beta$ & $(-1.07228,4.83052)$ & $(2.43012,2.85061)$ & $(1.90212,2.75121)$ & $(1.95853,2.56552)$ \\
$\lambda$ & $(0.800404,3.30531)$ & $(2.1234,2.9451)$ & $(1.9871,2.3894)$ & $(1.9339,2.09541)$ \\
$S(t)$ & $(0.727598,1.01213)$ & $(0.7542,0.9978)$ & $(0.7234,0.8754)$ & $(0.875696,0.90264)$ \\
$h(t)$ & $(0.0631523,0.460017)$ & $(0.2543,0.4234)$ & $(0.1653,0.3572)$ & $(0.200505,0.250584)$ \\
\hline
\end{tabular}

TABLE 4: MSE of ML and Bayesian estimates for the parameter $\alpha$ with $\alpha_{\circ}=1$.

\begin{tabular}{|c|c|c|c|c|c|}
\hline \multirow{2}{*}{$(n, m)$} & \multirow{2}{*}{ CS } & \multirow{2}{*}{ MLE } & \multirow{2}{*}{ SEL } & \multicolumn{2}{|c|}{ LINEX } \\
\hline & & & & $c=-2$ & $c=2$ \\
\hline \multirow{3}{*}{$(30,20)$} & I & $0.876(0.0453)$ & $0.866(0.0437)$ & $0.865(0.0431)$ & $0.866(0.0437)$ \\
\hline & II & $0.9292(0.0453)$ & $0.9243(0.0452)$ & $0.9254(0.0447)$ & $0.9131(0.0449)$ \\
\hline & III & $0.8468(0.0523)$ & $0.8399(0.0522)$ & $0.8355(0.0523)$ & $0.8329(0.0517)$ \\
\hline \multirow{3}{*}{$(40,20)$} & I & $0.8692(0.041)$ & $0.8681(0.041)$ & $0.8681(0.041)$ & $0.8681(0.041)$ \\
\hline & II & $0.8887(0.034)$ & $0.8873(0.0332)$ & $0.8573(0.0324)$ & $0.8858(0.0327)$ \\
\hline & III & $0.8858(0.0414)$ & $0.8853(0.0413)$ & $0.8845(0.0412)$ & $0.8849(0.0413)$ \\
\hline \multirow{3}{*}{$(40,30)$} & I & $0.8877(0.0481)$ & $0.8852(0.0481)$ & $0.8858(0.048)$ & $0.8845(0.048)$ \\
\hline & II & $0.9379(0.0485)$ & $0.9378(0.0471)$ & $0.9378(0.0473)$ & $0.9377(0.0472)$ \\
\hline & III & $0.9143(0.0483)$ & $0.9129(0.0482)$ & $0.9129(0.0481)$ & $0.9128(0.048)$ \\
\hline
\end{tabular}

\section{Simulation Study}

In order to compare the estimators of parameters and some lifetime parameters' reliability function and hazard function of the FRL-IL distribution, a simulation study was performed utilizing 1,000 progressive type-II censored samples for each simulation. To generate progressive type-II censored samples from the FRL-IL distribution, with initial values $\alpha_{\circ}=1, \beta_{\circ}=0.5$ and $\lambda_{\circ}=0.6$, the comparison of the different approaches of the estimators of the results $\alpha, \beta, \lambda, S(t)$ and $h(t)$, at $t=0.4$, has been considered in their mean square error (MSE), which is computed for $k=1,2,3,4,5\left(\psi_{1}=\alpha, \psi_{2}=\beta, \psi_{3}=\lambda, \psi_{4}=\right.$ $\left.S(0.4), \psi_{5}=h(0.4)\right) \quad$ and $\operatorname{MSE}\left(\psi_{k}\right)=(1 / M) \sum_{i=1}^{M}$ $\left(\widehat{\psi}_{k}^{(i)}-\psi_{k}\right)^{2}$, where $M=100$ is the number of simulated samples. Another criterion is used to compare the 95\% CIs obtained by using asymptotic distributions of the MLEs and CRIs. The comparison between them is made in terms of the average confidence interval lengths (ACLs) and coverage probability (CP). A confidence interval's $\mathrm{CP}$ is the percentage of time the interval contains the starting value of interest. The following progressive schemes are studied in this study:

Scheme I: $R_{1}=n-m, R_{i}=0$ for $i \neq 1$.

Scheme II: $R_{(m / 2)}=R_{(m / 2)+1}=((n-m) / 2), R_{i}=0$ for $i \neq(m / 2)$ and $i \neq(m / 2)+1$.

Scheme III: $R_{m}=n-m, R_{i}=0$ for $i \neq m$. 
TABLE 5: MSE of ML and Bayesian estimates for the parameter $\beta$ with $\beta_{0}=0.5$.

\begin{tabular}{lccccc}
\hline$(n, m)$ & CS & MLE & SEL & \multicolumn{2}{c}{ LINEX } \\
& & & & $c=-2$ & $0.751(0.1775)$ \\
$(30,20)$ & I & $0.7553(0.1976)$ & $0.752(0.1974)$ & $0.752(0.1975)$ & $0.6944(0.1229)$ \\
& II & $0.6947(0.123)$ & $0.6942(0.1228)$ & $0.7638(0.1723)$ & $0.6943(0.1229)$ \\
& III & $0.7641(0.1724)$ & $0.7639(0.1723)$ & $0.3343(0.6736)$ & $0.3343(0.6737)$ \\
$(40,20)$ & I & $0.3344(0.0374)$ & $0.3341(0.6736)$ & $0.3773(0.033)$ & $0.3772(0.0329)$ \\
& II & $0.3775(0.0331)$ & $0.3774(0.0329)$ & $0.4141(0.0306)$ & $0.4142(0.0307)$ \\
\hline \multirow{3}{*}{$(40,30)$} & III & $0.4143(0.0308)$ & $0.4142(0.0307)$ & $0.6372(0.1581)$ & $0.6372(0.1581)$ \\
& I & $0.6381(0.1582)$ & $0.6372(0.1581)$ & $0.6631(0.1307)$ & $0.6632(0.1305)$ \\
& II & $0.6643(0.1395)$ & $0.6632(0.1308)$ & $0.7434(0.1357)$ & $0.7434(0.1358)$ \\
\hline
\end{tabular}

TABLE 6: MSE of ML and Bayesian estimates for the parameter $\lambda$ with $\lambda_{0}=0.6$.

\begin{tabular}{lccccc}
\hline$(n, m)$ & CS & MLE & SEL & LINEX \\
& & & & $c=-2$ & $c=2$ \\
$(30,20)$ & I & $0.4455(0.1653)$ & $0.4453(0.1651)$ & $0.4452(0.1652)$ & $0.4453(0.1652)$ \\
& II & $0.43(0.1707)$ & $0.4278(0.1698)$ & $0.4285(0.1701)$ & $0.4285(0.1695)$ \\
& III & $0.5491(0.1802)$ & $0.5435(0.1773)$ & $0.5462(0.1786)$ & $0.5462(0.1756)$ \\
\hline \multirow{3}{*}{$(40,20)$} & I & $0.4844(0.1753)$ & $0.4843(0.1749)$ & $0.4843(0.1749)$ & $0.4843(0.1749)$ \\
& II & $0.5376(0.1822)$ & $0.5373(0.1815)$ & $0.5374(0.1812)$ & $0.5374(0.1811)$ \\
& III & $0.4973(0.1789)$ & $0.4944(0.1783)$ & $0.4941(0.1788)$ & $0.4941(0.1766)$ \\
\hline & I & $0.4704(0.1468)$ & $0.4695(0.1458)$ & $0.4694(0.1459)$ & $0.4695(0.1459)$ \\
& II & $0.5183(0.1871)$ & $0.5111(0.1861)$ & $0.5117(0.1862)$ & $0.5117(0.1863)$ \\
& III & $0.4772(0.2073)$ & $0.4765(0.2042)$ & $0.4766(0.2042)$ & $0.4766(0.2041)$ \\
\hline
\end{tabular}

TABLE 7: MSE of ML and Bayesian estimates for the $S(t)$ with $t=0.4$.

\begin{tabular}{|c|c|c|c|c|c|}
\hline \multirow{2}{*}{$(n, m)$} & \multirow{2}{*}{ CS } & \multirow{2}{*}{ MLE } & \multirow{2}{*}{ SEL } & \multicolumn{2}{|c|}{ LINEX } \\
\hline & & & & $c=-2$ & $c=2$ \\
\hline \multirow{3}{*}{$(30,20)$} & $\mathrm{I}$ & $0.6066(0.0084)$ & $0.6058(0.0082)$ & $0.606(0.0081)$ & $0.606(0.0082)$ \\
\hline & II & $0.6119(0.0048)$ & $0.6118(0.0047)$ & $0.6118(0.0047)$ & $0.6117(0.0047)$ \\
\hline & III & $0.6139(0.0068)$ & $0.6135(0.0064)$ & $0.6134(0.0064)$ & $0.6136(0.0064)$ \\
\hline \multirow{3}{*}{$(40,20)$} & I & $0.4323(0.0357)$ & $0.4322(0.0357)$ & $0.4322(0.0357)$ & $0.4322(0.0357)$ \\
\hline & II & $0.4607(0.0279)$ & $0.4606(0.0271)$ & $0.4606(0.0271)$ & $0.4605(0.0271)$ \\
\hline & III & $0.4838(0.0229)$ & $0.4862(0.0228)$ & $0.4869(0.0228)$ & $0.4869(0.0228)$ \\
\hline \multirow{3}{*}{$(40,30)$} & I & $0.6143(0.0048)$ & $0.6142(0.0043)$ & $0.6142(0.0043)$ & $0.6142(0.0043)$ \\
\hline & II & $0.6063(0.0039)$ & $0.6062(0.0038)$ & $0.6062(0.0038)$ & $0.6062(0.0038)$ \\
\hline & III & $0.6162(0.0046)$ & $0.6161(0.0045)$ & $0.6161(0.0049)$ & $0.6161(0.0043)$ \\
\hline
\end{tabular}

TABLE 8: MSE of ML and Bayesian estimates for the $h(t)$ with $t=0.4$.

\begin{tabular}{lccccc}
\hline$(n, m)$ & CS & MLE & SEL & \multicolumn{2}{c}{ LINEX } \\
& & & & $c=-2$ & $c=2$ \\
$(30,20)$ & I & $0.922(0.0514)$ & $0.9212(0.0522)$ & $0.921(0.0521)$ & $0.921(0.0522)$ \\
& II & $0.9326(0.0364)$ & $0.9321(0.0362)$ & $0.9322(0.0363)$ & $0.9322(0.0362)$ \\
& III & $0.9001(0.0412)$ & $0.8997(0.0411)$ & $0.8998(0.041)$ & $0.8998(0.0411)$ \\
$(40,20)$ & I & $1.3626(0.1806)$ & $1.3625(0.1805)$ & $1.3625(0.1805)$ & $1.3625(0.1805)$ \\
& II & $1.2988(0.1434)$ & $1.2987(0.1432)$ & $1.2396(0.1094)$ & $1.2987(0.1432)$ \\
& III & $1.2398(0.1098)$ & $1.2396(0.1091)$ & $0.9093(0.0331)$ & $0.9093(0.033)$ \\
$(40,30)$ & I & $0.9093(0.0334)$ & $0.9093(0.0332)$ & $0.0307)$ & $0.9473(0.0307)$ \\
& II & $0.9476(0.0309)$ & $0.9473(0.0307)$ & $0.9126(0.0284)$ & $0.9126(0.0287)$ \\
\hline
\end{tabular}


TABLE 9: ACL and CP of $95 \%$ CIs for the parameters $\alpha, \beta$, and $\lambda$.

\begin{tabular}{|c|c|c|c|c|c|c|c|}
\hline \multirow{2}{*}{$(n, m)$} & \multirow{2}{*}{ CS } & \multicolumn{2}{|c|}{$\alpha$} & \multicolumn{2}{|c|}{$\beta$} & \multicolumn{2}{|c|}{$\lambda$} \\
\hline & & MLE & MCMC & MLE & MCMC & MLE & MCMC \\
\hline \multirow{3}{*}{$(30,20)$} & I & $1.4965(0.9343)$ & $0.0009(0.9336)$ & $7.1223(0.9435)$ & $0.0047(0.9423)$ & $87.8(0.9321)$ & $0.019(0.9318)$ \\
\hline & II & $1.7465(0.9466)$ & $0.0012(0.9457)$ & $6.2708(0.9255)$ & $0.0043(0.9241)$ & $24.149(0.9588)$ & $0.0202(0.9401)$ \\
\hline & III & $1.398(0.9706)$ & $0.0009(0.9555)$ & $6.754(0.9514)$ & $0.0043(0.9458)$ & $37.966(0.9436)$ & $0.0189(0.9435)$ \\
\hline \multirow{3}{*}{$(40,20)$} & I & $1.3442(0.9614)$ & $0.0009(0.9483)$ & $1.833(0.9673)$ & $0.0012(0.9277)$ & $9.2238(0.9322)$ & $0.0096(0.9259)$ \\
\hline & II & $1.329(0.9461)$ & $0.0009(0.9413)$ & $1.993(0.9524)$ & $0.0014(0.9515)$ & $4.0414(0.942)$ & $0.0102(0.9363)$ \\
\hline & III & $1.4255(0.9747)$ & $0.0009(0.9508)$ & $3.1719(0.9369)$ & $0.0022(0.9326)$ & $15.892(0.9707)$ & $0.0136(0.9659)$ \\
\hline \multirow{3}{*}{$(40,30)$} & I & $1.6211(0.9489)$ & $0.001(0.9333)$ & $8.8516(0.965)$ & $0.005(0.939)$ & $27.4142(0.9693)$ & $0.023(0.9435)$ \\
\hline & II & $1.7899(0.9559)$ & $0.0012(0.9543)$ & $7.6806(0.9471)$ & $0.0049(0.9469)$ & $17.08(0.9663)$ & $0.0246(0.9251)$ \\
\hline & III & $1.455(0.9302)$ & $0.001(0.9222)$ & $5.377(0.9462)$ & $0.0039(0.943)$ & $51.065(0.9594)$ & $0.0138(0.9586)$ \\
\hline
\end{tabular}

TABLE 10: ACL and CP of $95 \%$ CIs for the parameters $S(t)$ and $h(t)$.

\begin{tabular}{|c|c|c|c|c|c|}
\hline \multirow{2}{*}{$(n, m)$} & \multirow{2}{*}{ CS } & \multicolumn{2}{|c|}{$S(t)$} & \multicolumn{2}{|c|}{$h(t)$} \\
\hline & & MLE & MCMC & MLE & MCMC \\
\hline \multirow{3}{*}{$(30,20)$} & I & $0.3432(0.9602)$ & $0.002(0.9601)$ & $0.974(0.9603)$ & $0.005(0.958)$ \\
\hline & II & $0.3104(0.9296)$ & $0.0022(0.927)$ & $0.9436(0.9507)$ & $0.0055(0.9421)$ \\
\hline & III & $0.2935(0.9576)$ & $0.0019(0.9661)$ & $0.8368(0.9631)$ & $0.0047(0.9429)$ \\
\hline \multirow{3}{*}{$(40,20)$} & I & $0.3482(0.9506)$ & $0.0014(0.9502)$ & $1.0584(0.9467)$ & $0.0035(0.9462)$ \\
\hline & II & $0.3158(0.9694)$ & $0.0014(0.9704)$ & $1.0082(0.9592)$ & $0.0034(0.9256)$ \\
\hline & III & $0.3098(0.9454)$ & $0.0019(0.9382)$ & $0.9819(0.9581)$ & $0.0048(0.9544)$ \\
\hline \multirow{3}{*}{$(40,30)$} & I & $0.3171(0.9637)$ & $0.0027(0.9504)$ & $0.8287(0.9667)$ & $0.0065(0.9567)$ \\
\hline & II & $0.2732(0.9617)$ & $0.0027(0.9325)$ & $0.994(0.9486)$ & $0.0068(0.9479)$ \\
\hline & III & $0.2537(0.9672)$ & $0.0018(0.9488)$ & $0.7269(0.9457)$ & $0.0042(0.939)$ \\
\hline
\end{tabular}

The results of estimate parameters and their MSE are shown in Tables 4-8, and the results of ACL and CP of $95 \%$ CIs are shown in Tables 9 and 10.

From the results, the following conclusions can be drawn:

(1) It is observed that from Tables 4-8, as sample size increases, the MSEs decrease and Bayes estimates have the smallest MSEs for $\alpha, \beta, \lambda, S(t)$ and $h(t)$. Hence, Bayes estimates perform better than the MLE methods in all cases considered.

(2) Bayes estimates under LINEX with $c=2$ provide better estimates in the sense of having smaller MSEs.

(3) For fixed values of the sample $n$ and effective sizes $m$, scheme I performs better than schemes II and III in the sense of having smaller MSEs.

(4) From Tables 9 and 10 for various sample sizes, observed failures, and schemes, it can be seen that the CRIs provide more accurate results than the ACIs.

\section{Conclusions}

The aim of this study is to develop various methods for estimating and deriving confidence intervals for parameters of the FRL-IL distribution under progressive type-II samples. The different estimation methods proposed in this study were used to calculate the time period (in months) to recovery for some patients with bladder cancer. Using asymptotic distributions and parametric bootstrap methods, the MLEs of the unknown parameters are obtained and different confidence intervals proposed. On the other hand, the estimation results were used to calculate the failure risk of these components. A simulation study was conducted to evaluate the quality of the proposed estimations, and this study showed that Bayesian methods have good performance in all different cases. In addition, scheme I performs better than schemes II and III in the sense of having smaller MSEs. Since the distribution of FRL-IL is well suited to the applied data, this study can effectively contribute to the study of failure analysis of patients with bladder cancer.

In future works, we are going to do an estimation of the parameters of the flexible reduced logarithmic-inverse Lomax distribution under adaptive progressive type-II censored data, and also, we will make the comparison to all types of censored algorithms that we applied.

\section{Data Availability}

The bladder cancer data used to support the findings of this study are included within the article.

\section{Conflicts of Interest}

The authors declare that they have no conflicts of interest.

\section{References}

[1] J. P. Klein and M. L. Moeschberger, Survival Analysis: Techniques for Censored and Truncated Data, Springer Science and Business Media, Berlin, Germany, 1997. 
[2] N. R. Mann, R. E. Schafer, and N. D. Singpurwalla, Methods for Statistical Analysis of Reliability and Life Data, John Wiley and Sons, New York, NY, USA, 1974.

[3] S. Eryilmaz and I. Bayramoglu, "Spacings, exceedances and concomitants in progressive type II censoring scheme," Journal of Statistical Planning and Inference, vol. 136, pp. 527-536, 2006.

[4] W. Q. Meeker and L. A. Escobar, Statistical Methods for Reliability Data, John Wiley and Sons, New York, NY, USA, 1998.

[5] N. Balakrishnan, "Progressive censoring methodology: an appraisal,” Test, vol. 16, pp. 211-259, 2007.

[6] N. Balakrishnan and E. Cramer, The Art of Progressive Censoring, Applications to Reliability and Quality, Springer, Berlin, Germany, 2014.

[7] S. K. Tse, C. Yang, and H. K. Yuen, "Statistical analysis of Weibull distributed lifetime data under type II progressive, censoring with binomial removals," Journal of Applied Statistics, vol. 27, no. 8, pp. 1033-1043, 2020.

[8] N. Balakrishnan and R. A. Sandhu, "A simple simulational algorithm for generating progressive type-II censored samples," The American Statistician, vol. 49, no. 2, pp. 229-230, 1995a.

[9] N. Balakrishnan and R. Aggarwala, Progressive Censoring: Theory, Methods and Applications, Birkhauser, Basel, Switzerland, 2000.

[10] R. Aggarwala and N. Balakrishnan, "Recurrence relations for single and product moments of progressive type-II right censored order statistics from exponential and truncated exponential distributions," Annals of the Institute of Statistical Mathematics, vol. 48, no. 4, pp. 757-771, 1996.

[11] N. Balakrishnan and R. A. Sandhu, "Best linear unbiased and maximum likelihood estimation for exponential distributions under general progressive type-II censored samples," The Indian Journal of Statistics, Series B, vol. 58, no. 1, pp. 1-9, 1996.

[12] R. Aggarwala and N. Balakrishnan, "Some properties of progressive censored order statistics from arbitrary and uniform distributions with applications to inference and simulation," Journal of Statistical Planning and Inference, vol. 70, pp. 35-49, 1998.

[13] G. C. Montanari, G. Mazzanti, M. Cacciari, and J. C. Fothergill, "Optimum estimators for the Weibull distribution from censored test data. Progressively-censored tests," IEEE Transactions on Dielectrics and Electrical Insulation, vol. 5, no. 2, pp. 157-164, 1998.

[14] N. Balakrishnan and N. Kannan, "Point and interval estimation for parameters of the logistic distribution based on progressively type-II censored samples," Advances in reliability, vol. 20, pp. 431-456, 2001.

[15] M. A. M. Ali Mousa and Z. F. Jaheen, "Statistical inference for the burr model based on progressively censored data," Computers and Mathematics with Applications, vol. 43, pp. 1441-1449, 2002a.

[16] M. A. M. Ali Mousa and Z. F. Jaheen, "Bayesian prediction for progressively censored data from the Burr model," Statistical Papers, vol. 43, pp. 587-593, 2002b.

[17] M. A. M. Ali Mousa and S. A. Al-Sagheer, "Bayesian prediction for progressively type-II censored data from the Rayleigh model," Communications in Statistics-Theory and Methods, vol. 34, no. 12, pp. 2353-2361, 2006.

[18] U. H. Salemi, S. Rezaei, and S. Nadarajah, "A-optimal and D-optimal censoring plans in progressively type-II right censored order statistics," Statistical Papers, vol. 60, 2017.
[19] X. Qin, J. Yu, and W. Gui, "Goodness-of-fit test for exponentiality based on spacings for general progressive Type-II censored data," Journal of Applied Statistics, vol. 49, pp. 1-22, 2020.

[20] K. Maiti and S. Kayal, "Estimating reliability characteristics of the log-logistic distribution under progressive censoring with two applications," Annals of Data Science, pp. 1-40, 2020.

[21] K. Maiti and S. Kayal, "Estimation of parameters and reliability characteristics for a generalized Rayleigh distribution under progressive type-II censored sample," Communications in Statistics-Simulation and Computation, vol. 50, no. 11, pp. 3669-3698, 2021.

[22] M. Saha and A. S. Yadav, "Estimation of the reliability characteristics by using classical and Bayesian methods of estimation for $x$ gamma distribution," Life Cycle Reliability and Safety Engineering, vol. 10, pp. 1-15, 2021.

[23] M. M. Buzaridah, D. A. Ramadan, and B. S. El-Desouky, "Flexible reduced logarithmic-inverse lomax distribution with application for bladder cancer," Open Journal of Modelling and Simulation, vol. 9, no. 4, pp. 351-369, 2021.

[24] E. A. Ahmed, "Estimation of some lifetime parameters of generalized Gompertz distribution under progressively typeII censored data," Applied Mathematical Modelling, vol. 39, no. 18, pp. 5567-5578, 2015.

[25] M. A. W. Mahmoud, D. A. Ramadan, and M. M. M. Mansour, "Estimation of lifetime parameters of the modified extended exponential distribution with application to a mechanical model," Communications in Statistics-Simulation and Computation, pp. 1-14, 2020.

[26] J. F. Lawless, Statistical Methods and Methods for Lifetime Data, John Wiley and Sons, New York, NY, USA, 1982.

[27] W. H. Greene, Econometric Analysis, Prentice Hall, Hoboken, NJ, USA, 4th edition, 2000.

[28] R. M. El-Sagheer, "Estimation of parameters of Weibull-Gamma distribution based on progressively censored data," Statistical Papers, vol. 59, no. 2, pp. 725-757, 2018.

[29] B. Efron, The Jackknife, the Bootstrap and Other Resampling PlansSIAM, Philadelphia, PA, USA, CBMS-NSF Regional Conference Series in Applied Mathematics, 1982.

[30] P. Hall, "Theoretical comparison of bootstrap confidence intervals," The Annals of Statistics, vol. 16, no. 3, pp. 927-953, 1988.

[31] H. R. Varian, "A Bayesian approach to real estate assessment," Studies in Bayesian Econometric and statistics: In Honor of Leonard J. Savage, pp. 195-208, North-Holland Publishing Company, Amsterdam, Netherlands, 1975.

[32] A. Zellner, "Bayesian estimation and prediction using asymmetric loss functions," Journal of the American Statistical Association, vol. 81, no. 394, pp. 446-451, 1986.

[33] E. A. Ahmed, "Estimation and prediction for the generalized inverted exponential distribution based on progressively firstfailure-censored data with application," Journal of Applied Statistics, vol. 44, no. 9, pp. 1576-1608, 2017.

[34] H. Panahi and S. Asadi, "On adaptive progressive hybrid censored burr type III distribution: application to the nano droplet dispersion data," Quality Technology and Quantitative Management, vol. 18, no. 2, pp. 179-201, 2021.

[35] T. A. Abushal, "Parametric inference of Akash distribution for type-II censoring with analyzing of relief times of patients," AIMS Mathematics, vol. 6, no. 10, pp. 10789-10801, 2021.

[36] E. T. Lee and J. Wang, Statistical Methods for Survival Data Analysis, John Wiley and Sons, New York, NY, USA, 2003. 